9. Sze-Tsen Hu, Proc. Cambridge Philos. Soc. vol. 43 (1947) pp. 314-320.

10. Hsein-Chung Wang, Proceedings Akademie van Wetenschappen, Amsterdam, vol. 50 (1947).

11. K. Reidemeister, Topologie der Polyeder, Leipzig, 1933.

12. S. Eilenberg, Ann. of Math. vol. 45 (1944) pp. 407-447.

13. - Fund. Math. vol. 32 (1939) pp. 167-175.

14. W. Hurewicz, Proceedings Akademie von Wetenschappen, Amsterdam, vol. 38 (1935) pp. 521-528.

15. K. Borsuk, Fund. Math. vol. 24 (1935) pp. 249-558.

Magdalen College, Oxford University

\title{
TOPOLOGICAL CHARACTERIZATION OF FIELDS WITH VALUATIONS
}

\section{DANIEL ZELINSKY ${ }^{1}$}

1. Introduction. A topological field is a (commutative) field which is also a topological space satisfying the separation axiom $T_{0}$, and in which addition, subtraction and multiplication are continuous, twovariable functions. For our purposes it is unnecessary to assume that division is continuous.

If $F$ is any field, topological or not, we define a (nonarchimedean) valuation of $F$ to be a function $v$ on $F$ to a linearly ordered group $\Gamma$ with the symbol 0 adjoined, such that

$$
\begin{aligned}
v(x y) & =v(x) v(y), \\
v(x+y) & \leqq \max [v(x), v(y)], \\
v(x) & =0 \text { if and only if } x=0,
\end{aligned}
$$

for all $x, y$ of $F$. It is understood that for every $\gamma$ of $\Gamma, 0<\gamma$ and $0 \gamma=\gamma 0$ $=0$. Such a valuation of a field defines a topology, with respect to which $F$ is a topological field, when we specify that the neighborhoods of 0 in $F$ are the sets $U(\gamma)=[x \in F \mid v(x)<\gamma]$, one for each $\gamma$ in $\Gamma$. If $F$ was a topological field to begin with and the topology defined by the valuation is the same as the original topology of $F$, we say that the valuation preserves the topology of $F$.

The question we intend to answer is, "Which topological fields have valuations preserving their topologies?"

Presented to the Society, April 17, 1948; received by the editors January 21, 1948.

${ }_{1}^{1}$ This paper was written while the author was a National Research Fellow in Mathematics. 
This problem was solved by Shafarevitch [3 ${ }^{2}$ and by Kaplansky [2] in the case where the assumption that $\Gamma$ is an ordered group is strengthened to " $\Gamma$ is the set of positive real numbers," and where the assumption (2) is weakened to $v(x+y) \leqq v(x)+v(y)$. Such a function $v$ we shall call an absolute value. For a topological field $F$ to have an absolute value preserving its topology, it is necessary and sufficient [2, Theorem 3$]$ that $F$ satisfy these two conditions:

(io) The set of nilpotent ${ }^{3}$ elements is open.

(ii) If $A \subset F$ is bounded away from zero, then $A^{-1}$ is bounded. ${ }^{3}$

For $F$ to have a valuation preserving its topology, condition (ii) is still necessary. However, condition ( $i_{0}$ ) is not; in fact, a field with a valuation may have no nilpotent elements besides 0 and yet the topology may be not discrete. Condition $\left(i_{0}\right)$ essentially singled out the nilpotent elements for Shafarevitch and Kaplansky as the elements whose values are to be smaller than the identity element of $\Gamma$. But with our more general $\Gamma$, the set of elements of value smaller than the identity is not uniquely determined by the topological field $F$. What is the same thing, inequivalent valuations may give the same topology. Hence we are forced to replace $\left(i_{0}\right)$ by a condition on subsets of $F$ rather than on elements. We choose this existence condition:

(i) Some neighborhood of zero generates an additive group which is bounded.

It is easily seen that (i) is equivalent to the condition:

There is a bounded, open, additive subgroup of $F$.

With the aid of Theorem 1, it is equally easy to see that the following is also an equivalent condition:

There is a set of group-neighborhoods of zero in $F$ and some open subset of $F$ is bounded. ${ }^{4}$

We shall show that a topological field $F$ has a valuation preserving its topology if and only if $F$ satisfies (i) and (ii). We shall see in the course of the proof that fields satisfying (i) alone are plentiful and that the topology in such a field is closely linked to the arithmetic properties of the field (see Theorem 1 and its corollary).

${ }^{2}$ Numbers in brackets refer to the references cited at the end of the paper.

${ }^{3}$ An element $x$ is called nilpotent if $\lim _{n=\infty} x^{n}=0$. If $A C F$ then " $A$ is bounded away from zero" means " $A$ is disjunct from some neighborhood of zero." By $A^{-1}$ we denote the set of inverses of elements of $A$. And $A$ is said to be bounded in case, for every open set $V$ containing 0 , there is an open set $U$ about 0 such that $A U C V$. Here and elsewhere the product of two subsets of a field is the set of all products of two elements, one from each of the given subsets.

${ }^{4}$ The referee has pointed out that the existence of an open, bounded subset, that is, local boundedness, follows from (ii); and that our main theorem could also be proved by first exploiting (ii) and using (i) only in the final stages. 
Some preliminary remarks on boundedness of subsets of $F$ may be in order. In a field with a valuation or an absolute value, a set is bounded exactly when it is bounded in the usual sense: the values of its elements are bounded above. It follows, then, in well known fashion that an absolute value is a valuation (that is, is nonarchimedean) if and only if the additive group generated by 1 is bounded. In general, every subset of a bounded set is bounded. And, in our definition of boundedness, the notion is unchanged if we change the range of the variables $U$ or $V$ or both from the class of open sets about zero to an equivalent set of neighborhoods of zero. Every discrete field is itself bounded (in fact, these are the only bounded fields) and so conditions (i) and (ii) are satisfied. But such a field has a trivial valuation preserving its trivial topology: $v(x)=1$ if $x \neq 0, v(0)=0$, so that all our theorems will be true for discrete fields.

2. Condition (i) alone. Let $F$ be a topological field satisfying (i). There is an open, bounded, additive subgroup of $F$ which contains 1. For there is an open, bounded, additive subgroup $G$ of $F$ which is not $\{0\}$; if $b$ is a nonzero element of $G$, then $b^{-1} G$ is the required group.

There is an open, bounded subring $R$ of $F$ containing 1 . For if $G$ is an open, bounded, additive group containing 1 , let $R$ be $[a \in F \mid G a \subset G]$. Clearly $R$ is a ring and contains 1 . Since $G$ is both open and bounded, there is some neighborhood $U$ of zero such that $G U \subset G$. That is, $U \subset R$ and $R$ is open. Since $1 \in G, R \subset G$ and $R$ is bounded.

If $F$ is not discrete, any open, bounded subring of $F$ which contains 1 is an order..$^{5}$ For if $0 \neq a \in F$, then $R a$ is an open set about zero. Since $R$ is bounded there is a neighborhood $U$ of zero such that $R U \subset R a$. Choose an element $b \in R \cap U$ with $b \neq 0$ and have $b \in R b \subset R a, b=c a$, $a=c^{-1} b$, with $b$ and $c$ in $R$.

It is now clear that (i) is equivalent to the existence of a bounded, open order in $F$. Let us temporarily turn our attention to fields and their orders.

If $F$ is a field and $R$ is an order in $F$, we may topologize $F$ by designating the principal $R$-ideals as neighborhoods of zero. Whether or not we include fractional ideals and whether or not we limit ourselves to principal ideals makes no real difference. The one restriction we do make is that $\{0\}$ is not to be counted as an $R$-ideal unless $R=F$. It is easy to check that this designation makes $F$ a topological field.

On the other hand, $R$ can be used to define a "semivaluation" in

${ }^{5}$ An order in $F$ is a subring of $F$ which contains 1 and whose quotient field is $F$. 
the usual fashion: If $N$ is the multiplicative group of units of $R$ and if $F^{*}$ is the multiplicative group of nonzero elements of $F$, then we may order $F^{*} / N$ by writing $f N \leqq g N$ when $f g^{-1} \in R$. This makes $F^{*} / N$ a partially ordered group or $o$-group in the sense of Everett and Ulam $[1],{ }^{6}$ and the natural homomorphism $w$ of $F^{*}$ onto $F^{*} / N$ has the following properties:

$$
\begin{aligned}
w(x y) & =w(x) w(y), \\
w(x-y) & \leqq \max [w(x), w(y)] .
\end{aligned}
$$

Of course, (5) is to be interpreted to mean: If $\delta \in F^{*} / N$ and $\delta \geqq w(x)$, $w(y)$, then $\delta \geqq w(x-y)$. We usually adjoin the symbol 0 to $F^{*} / N$ with the provision that $0 \delta=\delta 0=0,0<\delta$ for all $\delta \in F^{*} / N$. Then (4) and (5) are still true when we extend $w$ by defining $w(0)=0$, and

$$
w(x)=0 \text { if and only if } x=0 .
$$

Any function $w$ that maps $F$ onto an $o$-group $\Delta$ with 0 adjoined and satisfies (4), (5) and (6) will be called a semivaluation. (Note that the only difference between a valuation and a semivaluation is that the group of values of the latter need not be linearly ordered.) Not only does each order in $F$ define a semivaluation, but each semivaluation $w$ defines an order, namely, $[x \in F \mid w(x) \leqq \epsilon]$, where $\epsilon$ is the identity element in $\Delta$. This is actually a one-to-one correspondence between orders in $F$ and semivaluations of $F$, but we shall omit the proofs.

Every semivaluation of $F$ defines a topology in $F$ exactly as a valuation would. Specifically, for each $\delta \in \Delta$, define a neighborhood of zero in $F$ by $[x \in F \mid w(x)<\delta]$. It is a straightforward job to prove that this definition converts $F$ into a topological field whose topology is the same as that defined by the order corresponding to the semivaluation $w$.

Thus in a field the orders are in one-to-one correspondence with the semivaluations and a topology may be defined either by an order or by the corresponding semivaluation. "Close to zero" in such a topology means "divisible by many elements of the order." It may also be of interest to point out that the resultant topologies are not in one-to-one correspondence with the orders in the field, but rather with the classes of equivalent orders. ${ }^{7}$

${ }^{6}$ An $o$-group is a group which is also a partially ordered set with respect to a relation, $\leqq$, with the further properties: If $a \leqq b$, then $a c \leqq b c$; and for any $a, b$, there is an element $c$ with $a \leqq c, b \leqq c$.

7 Two orders $R$ and $S$ are equivalent in case $R C a S$ and $S C b R$ for some nonzero elements $a$ and $b$ in $F$. 
Theorem 1. The following conditions on an order $R$ in a topological field $F$ are equivalent.

(7) The topology defined by $R$ is the same as that of $F$.

(8) The semivaluation corresponding to $R$ preserves the topology of $F$.

(9) The order $R$ is bounded and open.

Proof. We have already remarked that $R$ and its semivaluation define the same topology in $F$. Hence (7) is equivalent to (8). Since $R$ is bounded and open in the topology it defines, (7) implies (9). Lastly, if $R$ is open, every $R$-ideal $R a$ is open. But since $R$ is also bounded, every open set $V$ about zero contains $R U$ for a suitable open set $U$ containing 0 ; hence $V$ contains the $R$-ideal $R a$ where $a \in R \cap U$ and where $a \neq 0$ when $R \neq F$. This proves Theorem 1 .

CoROLlary. Each of the following conditions on a topological field $F$ is equivalent to condition (i).

(i') There is bounded, open order in $F$.

$\left(i^{\prime \prime}\right)$ There is an order in $F$ which defines the same topology as that of $F$.

$\left(i^{\prime \prime \prime}\right)$ There is a semivaluation of $F$ preserving the topology of $F$.

3. Conditions (i) and (ii). We now prove our principal theorem.

Theorem 2. A topological field $F$ has a valuation preserving its topology if and only if $F$ satisfies (i) and (ii).

Proof. Necessity is obvious. To prove sufficiency, let $R$ be any open, bounded order in $F$, and define $X$ to be $[x \in F \mid x \notin R$ and $\left.x^{-1} \notin R\right]$.

The set $X$ is bounded. For $X^{-1}=X$ is disjunct from $R$ which is a neighborhood of zero.

If we assume condition (i), a set $A$ is bounded exactly when $A \subset R b$ for some $b \neq 0$ in $F$. For there is some $R$-ideal $R c$ such that $A R c \subset R$, and we may choose $b=c^{-1}$, except when $R=F$ in which case any nonzero $b$ will do. We shall call such an element $b$ a bound for $A$. If $b$ is a bound for $A$, so is $b c^{-1}$ for any nonzero $c$ in $R$. Hence we may choose a bound $p$ for $X$ with the property that $p^{-1} \in R$, and define $Y$ to be the set of all $p^{n}(n=1,2, \cdots)$.

If $Y$ is not bounded then $\lim _{n=\infty} p^{-n}=0$. For, because of (ii), $Y^{-1}$ cannot be bounded away from zero, every neighborhood $R a$ of zero contains an element $p^{-k}$. But if $k^{\prime} \geqq k$ then $p^{-k^{\prime}} \in R p^{-k} \subset R a$. Thus $\lim p^{-n}=0$.

If $Y$ is not bounded, the set of nilpotent elements in $F$ is open. For it contains $R p^{-1}$ which is a neighborhood of zero, and contains the open set $R q$ about $q$ whenever it contains a nonzero element $q$. 
If $Y$ is not bounded, Theorem 2 is true. For Kaplansky's theorem [ 2 , Theorem 3] asserts that $F$ has an absolute value preserving its topology. This absolute value is in fact a valuation since the additive group generated by 1 , being a subset of $R$, is bounded.

If $Y$ is bounded we shall produce a valuation. Let $S$ be the ring generated by $R$ and $X$, that is, the set of all polynomials in elements of $X$ with coefficients in $R$.

If $Y$ is bounded, $S$ is an open, bounded order. Since $S$ contains $R$, $S$ is clearly an open order. But if $a \prod_{i=1}^{n} x_{i}$ is any monomial in $S$ with $a \in R$ and $x_{i} \in X$, then $a \prod x_{i} \in R(R p)^{n}=R p^{n} \subset R q$, where $q$ is a bound for $Y$. Since $R q$ is closed under addition, $S \subset R q$.

If $Y$ is bounded, Theorem 2 is true. The required valuation is the semivaluation $v$ corresponding to the order $S$. By Theorem 1, this semivaluation preserves the topology of $F$. It only remains to show that $v$ is a valuation, that is, that $v\left(F^{*}\right)$ is linearly ordered. This follows from an obvious property of $S$ : if $x \in F$ then $x \in S$ or $x^{-1} \in S$. For if $x$ and $y$ are nonzero elements of $F$, then either $x^{-1} y \in S$ or $y^{-1} x \in S$. That is, either $v(y) \leqq v(x)$ or $v(x) \leqq v(y)$. This completes the proof.

\section{REFERENCES}

1. C. J. Everett and S. Ulam, On ordered groups, Trans. Amer. Math. Soc. vol. 57 (1945) pp. 208-216.

2. I. Kaplansky, Topological methods in valuation theory, Duke Math. J. vol. 14 (1947) pp. 527-541.

3. I. Shafarevitch, On the normalizability of topological fields, C. R. (Doklady) Acad. Sci. URSS. vol. 40 (1943) pp. 133-135.

The Institute for Advanced Study 